\title{
CF290 for pancolonic chromoendoscopy improved sessile serrated polyp detection and procedure time: a propensity score-matching study
}

다 (우우

\author{
Authors \\ Institutions \\ 1 Gastroenterology, Toyoshima Endoscopy Clinic, Tokyo- \\ Setagaya 157-0066, Japan \\ 2 Department of Gastroenterology, Graduate School of \\ Medicine, The University of Tokyo, Tokyo-Bunkyo, Japan \\ 3 Gastroenterology, Tokyo Medical Center, Tokyo- \\ Meguro, Japan \\ 4 Gastroenterology, Sakitani Endoscopy Clinic, Chiba- \\ Tsudanuma, Japan \\ 5 Department of Surgical Oncology, Graduate School of \\ Medicine, The University of Tokyo, Tokyo-Bunkyo, Japan \\ 6 Pathology and Cytology Laboratory Japan, Tokyo- \\ Suginami, Japan
}

Osamu Toyoshima1, Shuntaro Yoshida ${ }^{1,2}$, Toshihiro Nishizawa ${ }^{1,3}$, Tadahiro Yamakawa ${ }^{1}$, Kosuke Sakitani ${ }^{1,4}$, Keisuke Hata $^{1,5}$, Yoshiyuki Takahashi ${ }^{1}$, Mitsuhiro Fujishiro ${ }^{2}$, Hidenobu Watanabe ${ }^{6}$, Kazuhiko Koike ${ }^{2}$

submitted 22.11.2018

accepted after revision 9.4.2019

Bibliography

DOI https://doi.org/10.1055/a-0953-1909 |

Endoscopy International Open 2019; 07: E987-E993

(c) Georg Thieme Verlag KG Stuttgart · New York

elSSN 2196-9736

Corresponding author

Osamu Toyoshima, MD, Director, Gastroenterology,

Toyoshima Endoscopy Clinic, 6-17-5 Seijo, Setagaya-ku,

Tokyo 157-0066, Japan

Fax: +81-3-5429-9511

t@ichou.com

\section{ABSTRACT}

Background and study aims The benefits of the newgeneration CF290 (Olympus, Tokyo, Japan) for pancolonic chromoendoscopy (PCC) for colorectal polyp detection and its procedure time remain questionable. We compared the CF290 with the previous CF260 for PCC.

Methods We performed a propensity score-matching study using baseline characteristics such as age, sex, indications, endoscopist, and bowel preparation. We compared the detection of adenomas and sessile serrated polyps (SSPs) and procedure times of two expert endoscopists who performed PCC using the CF290 series (high-quality system with flushing pump) and the CF260 series (high-definition system).

Results We matched 374 patients who underwent PCC using the CF290 and 187 patients who underwent PCC using the CF260. The adenoma detection rate of the 290 series was higher than that of the 260 series, but not significantly. The SSP detection rate for the 290 series was higher than that for the 260 series $(P=0.01)$. Insertion time required for the 290 series was shorter than that required for the 260 series $(P<0.0001)$. Withdrawal time of the 290 series was shorter than that of the 260 series $(P<0.0001)$.

Conclusion Advanced technology can provide accuracy and help save time, and therefore, should be applied whenever possible.

\section{Introduction}

Colorectal cancer (CRC) is the third most common cancer worldwide. Removal of adenoma prevents CRC [1 -3]; however, a colonoscopy can miss adenoma, including advanced adenoma and interval cancer. The adenoma detection rate (ADR) indicates the quality of the colonoscopy [2-4], and different image-enhancing modalities have improved ADR. One of these modalities is pancolonic chromoendoscopy (PCC). PCC has been reported to improve ADR [5]. One previous report de- scribed that ADR was higher and that more adenomas per patient were found in the high-definition PCC group than that in the high-definition white light colonoscopy group [6]. It was also reported that PCC detected more flat adenomas and adenomas smaller than $5 \mathrm{~mm}$ in diameter per patient.

Changes in the function of a new-generation endoscope (290 series; Olympus, Tokyo, Japan) are described in this work. Visibility has improved with the new system because the amount of light has increased with new lenses and mirrors, and because the viewing angle has been widened from 140 de- 

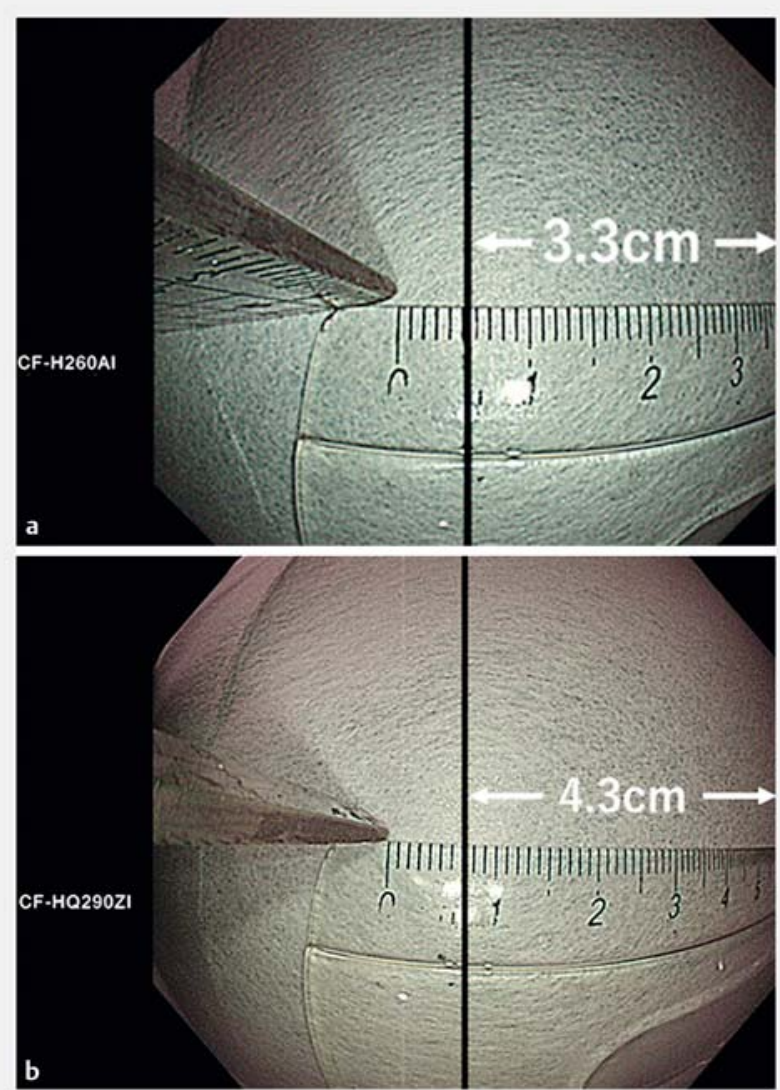

- Fig. 1 Image of the widened view angle. a View of the CF-H260AI. Distance from the tip of the endoscope to the subject, $3.0 \mathrm{~cm}$; distance from the center to the right end, $3.3 \mathrm{~cm}$. b View of the CFHQ290ZI. Distance from the tip of the endoscope to the subject, $3.0 \mathrm{~cm}$; distance from the center to the right end, $4.3 \mathrm{~cm}$.

grees to 170 degrees ( $\triangleright$ Fig. 1 ) [7]. It is equipped with a responsive insertion technology. A passive bending section, which is on the proximal side of the main bending section of the colonoscope, bends automatically in response to the force of the main bending section of the colon wall ( $\triangleright$ Fig. 2). The new flushing pump system can send water directly through the scope, not through the colonoscope accessory channel, into the colonic lumen. The Olympus 290 and Olympus 260 series are usually used in Japan and Europe; in addition, the Olympus 190 and Olympus 160 series are used in Europe and North America. The Olympus 290 and Olympus 260 series use the field-sequential color system, whereas the Olympus 190 and Olympus 160 series use the field-simultaneous color system. The 290 series is equivalent to the 190 series, and the 260 series is equivalent to the 160 series.

Few studies on detection of colorectal polyps and efficiency of the procedure have focused specifically on the new-generation endoscopes for PCC. In this work, we described the first study that tested whether the Olympus 290 series endoscope improves detection of polyps during a routine colonoscopy.

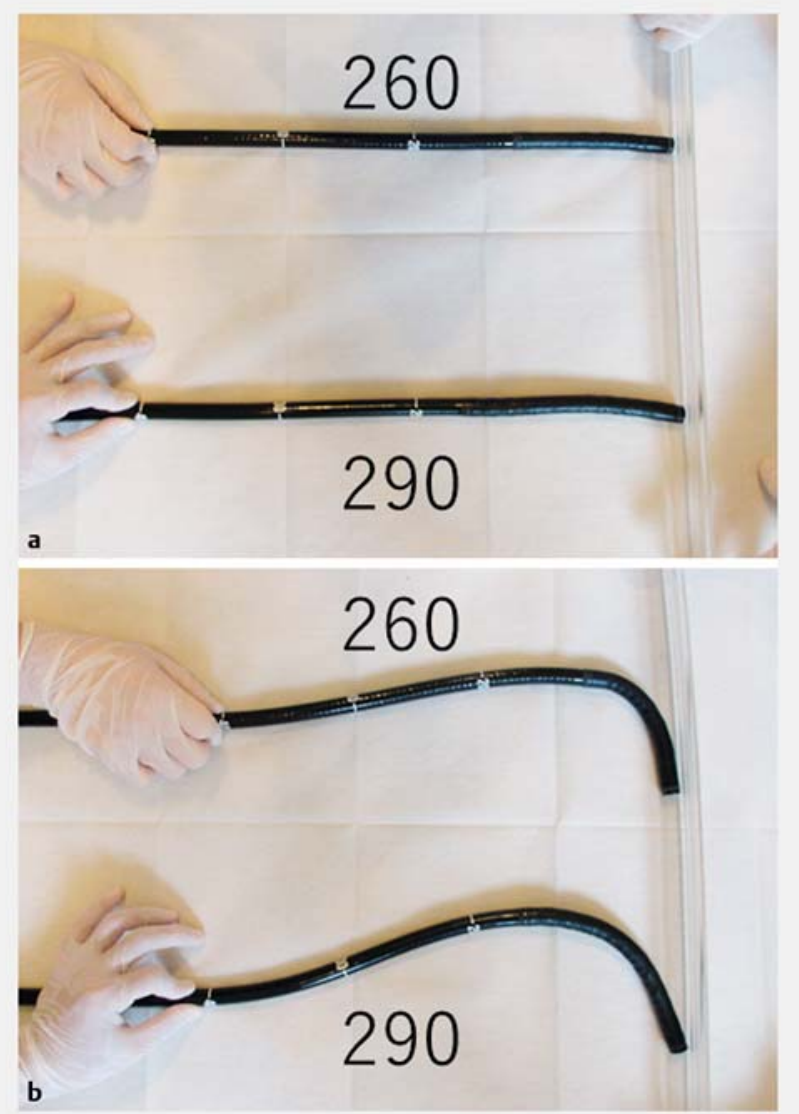

- Fig. 2 Image of responsive insertion technology. a Neutral position of the CF-H260Al and that of the CF-HQ290ZI. b Pressure was applied to the scopes. We held the part $40 \mathrm{~cm}$ from the tip of the scopes and moved the scopes $5 \mathrm{~cm}$ toward the wall. The CF290 bent more from the proximal side than the CF260.

\section{Methods}

\section{Ethics}

This retrospective study was approved by the Ethical Review Committee of the Hattori Clinic on September 7, 2017. Written informed consent was obtained from the participants. All clinical investigations were conducted according to the ethical guidelines of the Declaration of Helsinki.

\section{Study population}

This propensity score-matching study comprised subjects who agreed to participate in the study and underwent colonoscopy at Toyoshima Endoscopy Clinic, an outpatient clinic specializing in endoscopy, between June and December 2012 using the Olympus CF260 series and between April and August 2017 using the Olympus CF290 series. Colonoscopy was performed to evaluate symptoms (i.e., bleeding including positive results of fecal occult blood test, abdominal symptoms, and abnormal bowel habits), for screening, or for polyp surveillance. We excluded patients who had advanced CRC, those who previously had CRC or inflammatory bowel disease, those with indications 
for treatment including colorectal polypectomy and hemostasis, and those with poor bowel preparation precluding complete observation $[6,8]$.

\section{Colonoscopy equipment}

The CF260 group patients underwent colonoscopy using a high-definition endoscopy system with a high-definition colonoscope (CV-260 HiVision and CLV-260 with CF-H260Al, Evis Lucera Spectrum system; Olympus). Elite CF290 group patients underwent colonoscopy using a new-generation endoscopy system with a new-generation colonoscope (CV-290 and CLV290, with CF-HQ290ZI, CF-HQ290I, or PCF-H290ZI, Evis Lucera Elite system; Olympus), a new-generation flushing pump (OFP2; Olympus), and a carbon dioxide $\left(\mathrm{CO}_{2}\right)$ insufflator (UCR $\mathrm{CO}_{2}$ regulation unit; Olympus). PCF-H290ZI was used for patients aged 80 years or older, patients aged 70 years or older and who had undergone a previous abdominal surgery, and patients with difficult insertion due to a colon adhesion found during a previous colonoscopy. Narrow band imaging (NBI) is available for both the 260 series and 290 series.

\section{Colonoscopy procedure}

All colonoscopies were performed by two experienced boardcertificated attending endoscopists and gastroenterologists who had performed more than 10,000 colonoscopies.

Colonoscope insertion in the cecum was accomplished using standard maneuvers. Small shaking, jiggling, and right-turn shortening maneuvers have been frequently used for insertion [9]. We started the colonoscopy with the patients in the left lateral position. Then, we placed the patients in the supine position after observation of the lower rectum. Extra gas and liquid were aspirated and removed as much as possible. We conducted colonoscopies using water-aided techniques with a $20-\mathrm{mL}$ syringe placed directly through the colonoscope accessory channel for patients in the 260 group; we used the OFP- 2 for patients in the 290 group [10]. For colonic insufflation, air was used for patients in the 260 group and patients in the 290 group with chronic respiratory failure; $\mathrm{CO}_{2}$ was administered through the UCR for patients in the 290 group without chronic respiratory failure.

Colonoscopies were performed under conscious sedation with midazolam $(0.5$ to $10 \mathrm{mg})$ and/or pethidine hydrochloride $(17.5$ to $70 \mathrm{mg})$. In the absence of contraindications, when the colonoscope reached the cecum, we administered 10 to $20 \mathrm{mg}$ of scopolamine butylbromide.

Observation time for withdrawal of the colonoscope was standardized as at least 6 minutes [11]. PCC involved pan-colonic spraying with $0.05 \%$ indigo carmine by a $20-\mathrm{mL}$ syringe placed directly through the colonoscope accessory channel. We repeated administration of $5 \mathrm{~mL}$ indigo carmine with $10 \mathrm{~mL}$ air-spraying 10 to 20 times during the withdrawal procedure, and pools of excess dye were suctioned before examination. The colonoscope was sequentially withdrawn as the prescribed position changed $[12,13]$ : the ascending colon/hepatic flexure was examined in the left lateral decubitus position; the transverse colon was examined in the supine position; the splenic flexure, descending colon, and sigmoid-descending colon junc- tion were examined in the right lateral position; and the sigmoid colon and rectum were examined in the right lateral position. Physicians were permitted to change the position if it was medically necessary.

To endoscopically diagnose colorectal polyps, we used the updated Paris Endoscopic Classification of superficial neoplastic lesions in the digestive tract and the NICE (NBI International Colorectal Endoscopic) classification $[14,15]$. Lesions diagnosed as adenomas or sessile serrated polyps (SSPs) were removed by using hot or cold polypectomy with a snare or forceps or by endoscopic mucosal resection on the examination day. We did not resect the polyps with a diameter of $20 \mathrm{~mm}$ or more because they should be resected in the hospitalization facility.

Patients involved in this study underwent colonic preparation using $2 \mathrm{~L}$ of polyethylene glycol solution administered orally 5 hours before the procedure. Polyethylene glycol solution or magnesium citrate was added when the stool was not clear liquid. Quality of the bowel preparation was graded as A (all colon segments empty and clean or minor amount of fluid in the gut that was easily removed by suction), B (at least one colon segment with residual amounts of brown liquid or semi-solid stool that could be easily removed or displaced), C (at least one colon segment with only partially removable stool preventing complete visualization of mucosa), or D (at least one colon segment that could not be examined due to presence of remaining solid stool). The following colon segments were rated: rectum, sigmoid colon, descending colon, transverse colon, and ascending colon/cecum [16]. Patients with a grade of D during colonoscopy were excluded.

\section{Diagnosis of polyps}

All resected specimens were examined histologically by hematoxylin and eosin staining. One experienced gastrointestinal pathologist diagnosed polyps, including adenomas and SSPs, according to World Health Organization criteria [17]. Traditional serrated adenomas were included in the adenoma category, but SSPs were not. An advanced adenoma was defined as an adenoma with a villous component, size larger than $10 \mathrm{~mm}$, or high-grade dysplasia based on the World Health Organization definition [17]. Only lesions that were histologically confirmed as adenomas or SSPs were counted [18].

\section{Statistical analysis}

We considered baseline patient characteristics, including age, sex, body mass index, smoking (Brinkman score $\geq 400$ ), drinking ( $\geq 1$ cups of alcohol per day), first-degree relative with a history of CRC, indications for colonoscopy (evaluation of symptoms, screening, or polyp surveillance), the colonoscopist who performed the procedure, and bowel preparation grade as potential confounding factors $[6,7,11,16,19-21]$. To reduce the effect of selection bias and potential confounding in this study, we performed rigorous adjustments for all baseline characteristics described above using propensity score-matching [22,23]. Patients who underwent colonoscopy with the CF290 series were identified and propensity score-matched with those who underwent colonoscopy with the CF260 series. Matching was 
- Table 1 Baseline characteristics of the patients before and after propensity score-matching.

\begin{tabular}{|c|c|c|c|c|c|c|}
\hline & \multicolumn{3}{|c|}{ Before matching } & \multicolumn{3}{|c|}{ After matching } \\
\hline & $\begin{array}{l}260 \text { series } \\
\mathrm{N}=\mathbf{2 1 6}\end{array}$ & $\begin{array}{l}290 \text { series } \\
\mathrm{N}=580\end{array}$ & $P$ value & $\begin{array}{l}260 \text { series } \\
N=187\end{array}$ & $\begin{array}{l}290 \text { series } \\
N=374\end{array}$ & $P$ value \\
\hline Mean age, years (SD) & $52.2(14.5)$ & $53.9(13.0)$ & 0.12 & $52.5(14.6)$ & $52.9(13.6)$ & 0.73 \\
\hline Male & $111(51.4 \%)$ & $266(45.8 \%)$ & 0.17 & $89(47.6 \%)$ & $175(46.8 \%)$ & 0.86 \\
\hline Mean BMI, $\mathrm{kg} / \mathrm{cm}^{2}(\mathrm{SD})$ & $22.0(3.1)$ & $22.1(3.3)$ & 0.73 & $22.0(3.1)$ & $21.9(3.2)$ & 0.68 \\
\hline Smoking & $23(10.6 \%)$ & $59(10.2 \%)$ & 0.84 & $19(10.2 \%)$ & $35(9.4 \%)$ & 0.76 \\
\hline Drinking & $53(24.5 \%)$ & $137(23.6 \%)$ & 0.79 & $51(27.3 \%)$ & $75(20.1 \%)$ & 0.054 \\
\hline Family history of CRC & $41(19.0 \%)$ & $89(15.3 \%)$ & 0.22 & $35(18.7 \%)$ & $65(17.4 \%)$ & 0.70 \\
\hline \multicolumn{7}{|l|}{ Indications } \\
\hline - Evaluation of symptoms & $53(24.5 \%)$ & $136(23.4 \%)$ & & $50(26.7 \%)$ & $93(24.9 \%)$ & \\
\hline - Screening & $90(41.7 \%)$ & $298(51.4 \%)$ & 0.21 & $79(42.2 \%)$ & $174(46.5 \%)$ & 0.45 \\
\hline - Polyp surveillance & $73(33.8 \%)$ & $146(25.2 \%)$ & 0.25 & $58(31.0 \%)$ & $107(28.6 \%)$ & 0.97 \\
\hline \multicolumn{7}{|l|}{ Endoscopist } \\
\hline - 1 & $190(88.0 \%)$ & $559(96.4 \%)$ & & 181 (96.8\%) & $359(96.0 \%)$ & \\
\hline .2 & $26(12.0 \%)$ & $21(3.6 \%)$ & $<0.0001$ & $6(3.2 \%)$ & $15(4.0 \%)$ & 0.64 \\
\hline \multicolumn{7}{|l|}{ Bowel preparation* } \\
\hline - A or B & $179(82.9 \%)$ & $516(89.0 \%)$ & & $161(86.1 \%)$ & $323(86.4 \%)$ & \\
\hline . C & $37(17.1 \%)$ & $64(11.0 \%)$ & 0.023 & $26(13.9 \%)$ & $51(13.6 \%)$ & 0.93 \\
\hline \multicolumn{7}{|c|}{$\begin{array}{l}\text { The caliper width was set to } 0.5878 \text {, the standard deviation of the logit function of the propensity score for patients who underwent colonoscopy with the CF260 } \\
\text { series of endoscope. P values were calculated by the Wald test using logistic regression. } \\
\text { * Bowel preparation: A, all colon segments empty and clean or minor amount of fluid in the gut, but easily removed by suction; B, at least one colon segment with } \\
\text { residual amounts of brown liquid or semi-solid stool that could be easily removed or displaced; C, at least one colon segment with only partially removable stool } \\
\text { preventing complete visualization of mucosa. BMI, body mass index; CRC, colorectal cancer; SD, standard deviation. }\end{array}$} \\
\hline
\end{tabular}

performed with a 1:2 matching protocol using nearest-neighbor matching without replacement and with a caliper width of 0.5878 , which was the standard deviation of the logit of the propensity score for patients who underwent colonoscopy with the CF260 series ( $\downarrow$ Table 1 ) [24-26].

After propensity score-matching was performed, we compared polyp detection rates, polyp numbers, insertion times, withdrawal times including the times required for polypectomy, and use of sedatives of the two procedure groups. We assessed $P$ values by using the Wald test with logistic regression.

Statistical significance was considered a two-sided $P<0.05$. All statistical analyses were performed using $R$ version 3.3.3 ( $R$ Core Team 2017; R Foundation for Statistical Computing, Vienna, Austria).

\section{Results}

\section{Study population}

We identified 796 individuals who met our inclusion criteria ( Fig.3); of these, 216 underwent colonoscopy using the CF260 series and 580 underwent colonoscopy using the CF290 series. Cecal intubation was achieved in all patients. Before propensity score-matching, there were differences in the baseline variables of endoscopist and preparation for the two groups ( $\triangleright$ Table 1). With use of propensity-score matching, 374 patients who underwent colonoscopy using the 290 series were matched with 187 patients who underwent colonoscopy using the 260 series. After matching, there was no longer any significant difference between the two groups with respect to any baseline characteristics.

\section{Outcomes for matched patients}

A comparison of outcomes of the matched patients in CF260 and CF290 series groups is shown in > Table 2 . The adenoma detection rate (ADR) of the 290 series group (46.3\%) was higher than that of the 260 series group (44.4\%), but not significantly. The number of adenomas in the 290 series group $(0.90$ per patient) was larger than that in 260 series group $(0.83$ per patient), but not significantly. A sub-analysis of different types of adenomas did not indicate a difference in the adenoma numbers. The SSP detection rate was higher in the 290 series group $(6.4 \%)$ than that in the 260 series group $(1.1 \% ; P=0.01)$. The number of SSPs was larger in the 290 series group $(0.078$ per patient) than that in the 260 series group $(0.011$ per patient; $P<0.01)$ 
- Table 2 Comparison of the outcomes of the 260 series group and 290 series group patients matched according to propensity scores.

\begin{tabular}{|c|c|c|c|c|}
\hline & $\begin{array}{l}260 \text { series } \\
\mathrm{N}=187\end{array}$ & $\begin{array}{l}290 \text { series } \\
N=374\end{array}$ & OR/Coefficient (95\% Cl) & $P$ value \\
\hline Patients with at least one adenoma & $83(44.4 \%)$ & $173(46.3 \%)$ & $1.1(0.76,1.5)$ & 0.67 \\
\hline No. of adenomas per patient ${ }^{1}$ & $0.83(1.3)$ & $0.90(1.4)$ & $0.070(-0.17,0.31)$ & 0.57 \\
\hline No. of cancers per patient ${ }^{1}$ & $0.005(0.073)$ & $0.005(0.073)$ & $-0.0(-0.013,0.013)$ & 1.00 \\
\hline No. of advanced adenomas per patient ${ }^{1}$ & $0.027(0.16)$ & $0.032(0.19)$ & $0.005(-0.027,0.037)$ & 0.74 \\
\hline No. of adenomas in the right colon per patient ${ }^{1}$ & $0.59(1.1)$ & $0.68(1.1)$ & $0.096(-0.099,0.29)$ & 0.34 \\
\hline No. of adenomas $\leq 5 \mathrm{~mm}$ per patient ${ }^{1}$ & $0.74(1.3)$ & $0.79(1.2)$ & $0.043(-0.17,0.26)$ & 0.70 \\
\hline No. of flat adenomas per patient ${ }^{1}$ & $0.61(1.1)$ & $0.81(1.3)$ & $0.20(-0.015,0.42)$ & 0.07 \\
\hline Patients with at least one sessile serrated polyp & $2(1.1 \%)$ & $24(6.4 \%)$ & $6.3(1.5,27)$ & 0.01 \\
\hline No. of sessile serrated polyps per patient ${ }^{1}$ & $0.011(0.15)$ & $0.078(0.31)$ & $0.067(0.019,0.11)$ & $<0.01$ \\
\hline Insertion time ${ }^{1}$, min & $5.9(5.1)$ & $4.1(1.9)$ & $-1.8(2.4,-1.2)$ & $<0.0001$ \\
\hline Withdrawal time ${ }^{1}$, min & $15.8(4.7)$ & $13.1(2.9)$ & $-2.8(3.4,-2.1)$ & $<0.0001$ \\
\hline Use of pethidine hydrochloride & $98(52.4 \%)$ & $173(46.3 \%)$ & $0.78(0.55,1.1)$ & 0.17 \\
\hline Use of midazolam & $163(87.2 \%)$ & $306(81.8 \%)$ & $0.66(0.40,1.1)$ & 0.11 \\
\hline
\end{tabular}

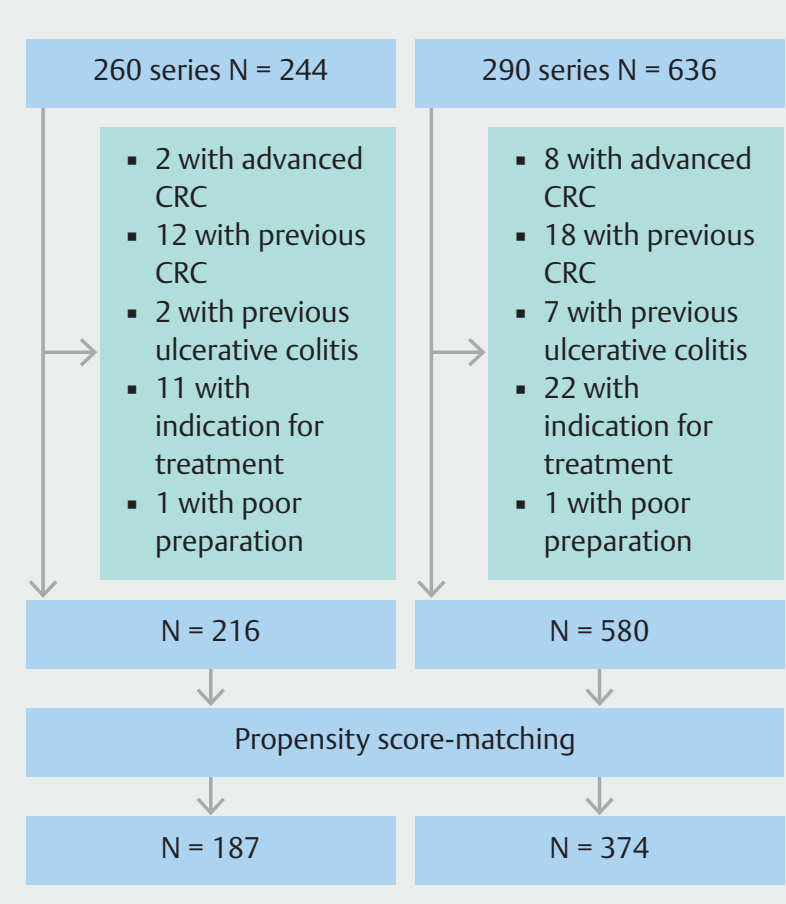

- Fig. 3 Flow chart of patient enrollment and propensity scorematching. CRC, colorectal cancer.

Mean insertion time of the 290 series group was 1.8 minutes shorter than that of the 260 series group $(P<0.0001)$. Mean withdrawal time of the 290 series group was 2.7 minutes short- er than that of 290 series group $(P<0.0001)$. Frequency of sedative use was not significantly different between the groups.

\section{Discussion}

We found that PCC using the new-generation endoscope resulted in a higher SSP detection rate and a larger number of SSP diagnosed than PCC using the previous endoscope. We found that the ADR and number of adenomas, especially flat adenomas, of those who underwent chromocolonoscopy using the 290 series were higher than those of patients who underwent chromocolonoscopy using the 260 series, but not significantly. Corley et al [3] reported that the ADR of the highest quintile was $33.5 \%$ to $52.5 \%$. Because colonoscopy using the previous endoscope had already achieved the highest quintile of the ADR, colonoscopy using the new-generation endoscope could not achieve a significantly higher detection rate. We considered several reasons why significant differences were obtained for SSP detection with a highly accurate colonoscopy. SSP can be difficult to detect because of their flat morphology and indistinct borders [27]. It is not unusual to see mucin fill the lumen of dilated crypts and coat the surface of the SSP. Colonoscopy using new-generation tools involved improved visibility provided by the widened viewing angle and increased light intensity, which might enable detection of some flat and/or indistinct polyps, especially in the right colon, which has deep folds. Furthermore, water-aided colonoscopy with the OFP-2 could effectively remove the mucus covering the polyps because of its sustained water supply. 
We demonstrated that the new-generation endoscope shortened insertion and withdrawal times, with a total procedure time of 5 minutes. Therefore, a colonoscopist who performs 1,200 colonoscopies annually can save 100 hours per year. Shortened insertion time seemed to be attributed to the responsive insertion technology. We also believe the increased light intensity and the widened viewing angle contributed to the shorter procedure times.

There are some limitations to our study. First, this study was a historical retrospective comparison. The 5-year differences between the two groups can have large implications on findings of this study include the learning curves of the endoscopists, changes in colonoscopy practice, and study population. Second, we did not assess the effects of improvements in visibility and operability, including the widened viewing angle, water supply function, passive bending section of the scope, and $\mathrm{CO}_{2}$ insufflation on ADR or procedure time. Third, it is difficult to exclude the endoscopist and pathologist recognition bias in SSP detection. Knowledge, awareness, and skills of identification of both the endoscopist and pathologist might have contributed to an increase in SSP detection rate. Studies should be planned to address these aspects and corroborate our findings. Fourth, propensity score model overfitting has been reported to lead to inflated variances of the estimated odds ratios $[28,29]$. Furthermore, adjustment using propensity score matching has recently been criticized as a paradoxically problematic method [30]. Propensity score-matching in our study might have resulted in some biases

\section{Conclusion}

We found that better technology improves accuracy and shortens procedural time. Thus, new technology should be used whenever possible.

\section{Competing interests}

Dr. Fujishiro has received personal fees from Olympus Corporation outside of the submitted work.

\section{References}

[1] Zauber AG, Winawer S], O'Brien M] et al. Colonoscopic polypectomy and long-term prevention of colorectal-cancer deaths. N Engl J Med 2012; 366: 687-696

[2] Nishihara R, Wu K, Lochhead P et al. Long-term colorectal-cancer incidence and mortality after lower endoscopy. N Engl J Med 2013; 369 : $1095-1105$

[3] Corley DA, Jensen CD, Marks AR et al. Adenoma detection rate and risk of colorectal cancer and death. N Engl J Med 2014; 370: 1298 1306

[4] Kaminski MF, Regula J, Kraszewska E et al. Quality indicators for colonoscopy and the risk of interval cancer. N Engl J Med 2010; 362: $1795-1803$

[5] Pohl J, Schneider A, Vogell H et al. Pancolonic chromoendoscopy with indigo carmine versus standard colonoscopy for detection of neo- plastic lesions: a randomised two-centre trial. Gut 2011; 60: 485 490

[6] Kahi C], Anderson JC, Waxman I et al. High-definition chromocolonoscopy vs. high-definition white light colonoscopy for average-risk colorectal cancer screening. Am J Gastroenterol 2010; 105: 1301 1307

[7] Leung WK, Lo OS, Liu KS et al. Detection of colorectal adenoma by narrow band imaging (HQ190) vs. high-definition white light colonoscopy: a randomized controlled trial. Am J Gastroenterol 2014; 109: $855-863$

[8] Lai EJ, Calderwood AH, Doros G et al. The Boston bowel preparation scale: a valid and reliable instrument for colonoscopy-oriented research. Gastrointest Endosc 2009; 69: 620-625

[9] Lee SH, Park YK, Lee DJ et al. Colonoscopy procedural skills and training for new beginners. World J Gastroenterol 2014; 20: 16984 16995

[10] Fuccio L, Frazzoni L, Hassan C et al. Water exchange colonoscopy increases adenoma detection rate: a systematic review with network meta-analysis of randomized controlled studies. Gastrointest Endosc 2018; 88: 589- 597

[11] Barclay RL, Vicari J], Doughty AS et al. Colonoscopic withdrawal times and adenoma detection during screening colonoscopy. N Engl J Med 2006; 355: $2533-2541$

[12] East JE, Suzuki N, Arebi $\mathrm{N}$ et al. Position changes improve visibility during colonoscope withdrawal: a randomized, blinded, crossover trial. Gastrointest Endosc 2007; 65: 263-269

[13] Ou G, Kim E, Lakzadeh P et al. A randomized controlled trial assessing the effect of prescribed patient position changes during colonoscope withdrawal on adenoma detection. Gastrointest Endosc 2014; 80: $277-283$

[14] Endoscopic Classification Review Group. Update on the Paris classification of superficial neoplastic lesions in the digestive tract. Endoscopy 2005; 37: $570-578$

[15] Hewett DG, Kaltenbach T, Sano Y et al. Validation of a simple classification system for endoscopic diagnosis of small colorectal polyps using narrow-band imaging. Gastroenterology 2012; 143: 599-607. e1

[16] Ell C, Fischbach W, Bronisch H] et al. Randomized trial of low-volume PEG solution versus standard PEG + electrolytes for bowel cleansing before colonoscopy. Am J Gastroenterol 2008; 103: 883-893

[17] Bosman FT, Carneiro F, Hruban RH et al. WHO Classification of Tumours of the Digestive System.4th edition Lyon: International Agency for Research on Cancer; 2010

[18] Lee T], Rees C], Blanks RG et al. Colonoscopic factors associated with adenoma detection in a national colorectal cancer screening program. Endoscopy 2014; 46: $203-211$

[19] Gerard DP, Foster DB, Raiser MW et al. Validation of a new bowel preparation scale for measuring colon cleansing for colonoscopy: the Chicago bowel preparation scale. Clin Transl Gastroenterol 2013; 4: e43

[20] Toyoshima O, Hata K, Yoshida S et al. New-generation chromoendoscopy may increase confidence in the DISCARD2 study. Gut 2018; 67: $1742-1743$

[21] Anderson JC, Butterly LF, Weiss JE et al. Providing data for serrated polyp detection rate benchmarks: an analysis of the New Hampshire Colonoscopy Registry. Gastrointest Endosc 2017; 85: 1188 - 1194

[22] Rosenbaum PR, Rubin DB. The central role of the propensity score in observational studies for causal effects. Biometrika 1983; 70: 41 - 55

[23] D'Agostino RB Jr. Propensity score methods for bias reduction in the comparison of a treatment to a non-randomized control group. Stat Med 1998; 17: $2265-2281$

[24] Normand ST, Landrum MB, Guadagnoli E et al. Validating recommendations for coronary angiography following acute myocardial infarc- 
tion in the elderly: a matched analysis using propensity scores. J Clin Epidemiol 2001; 54: 387-398

[25] Austin PC. Optimal caliper widths for propensity-score matching when estimating differences in means and differences in proportions in observational studies. Pharm Stat 2011; 10: 150-161

[26] Bangalore S, Guo Y, Samadashvili Z et al. Everolimus-eluting stents or bypass surgery for multivessel coronary disease. N Engl J Med 2015; 372: $1213-1222$

[27] Rex DK, Ahnen DJ, Baron JA et al. Serrated lesions of the colorectum: review and recommendations from an expert panel. Am J Gastroenterol 2012; 107: 1315-1330
[28] Sjolander A. Propensity scores and M-structures. Stat Med 2009; 28: $1416-1420$

[29] Schuster T, Lowe WK, Platt RW. Propensity score model overfitting led to inflated variance of estimated odds ratios. J Clin Epidemiol 2016; 80: $97-106$

[30] Huybrechts KF, Ripollone JE, Rothman KJ et al. Implications of the propensity score matching paradox in pharmacoepidemiology. Am J Epidemiol 2018; 187: 1951-1961 